\title{
Vibrational Relaxation Dynamics of Polyatomic Molecules in Solution
}

\author{
Anatolio Pigliucci and Eric Vauthey
}

\begin{abstract}
Ultrafast time-resolved fluorescence measurements have been carried out to investigate vibrational relaxation dynamics of perylene derivatives in solution. The early results obtained with cyanoperylene in acetonitrile are presented and discussed.
\end{abstract}

Keywords: Energy dissipation · Ultrafast spectroscopy $\cdot$ Vibrational cooling $\cdot$ Vibrational relaxation

The transformation of light into heat is a very efficient and widespread process experienced by many creatures on earth, from the lizard drowsing under the sun to the driver in his black car, on a summer day, dreaming of an air-conditioning system. The key phenomenon underlying this conversion is vibrational relaxation (VR). VR involves two processes: intramolecular vibrational redistribution (IVR) and vibrational cooling (VC). The absorption of light by a molecule can result in the population of an electronic excited state that is itself vibrationally excited. This vibrational energy is in general called excess excitation energy. Due to the restrictions imposed by the law of momentum conservation, only very specific 'Franck-Condon active' vibrational modes can be excited upon such a vibronic transition. However, vibrational energy does not remain concentrated in these modes but redistributes in the other modes. This is schematically illustrated in Fig. 1 for a triatomic molecule. Upon excitation with light at the right wavelength, only vibrational states of mode 1 are populated (state $(2,0,0)$ in Fig. 1). There are, however, five other isoenergetic states where the vibrational energy is distributed differently among the three modes. IVR corresponds to transitions from state $(2,0,0)$ to these states.

${ }^{*}$ Correspondence: A. Pigliucci

Physical Chemistry Department

Sciences II

University of Geneva

30, Quai Ernest Ansermet

CH-1211 Geneva 4

Tel.: +41227026551

E-Mail: anatolio.pigliucci@chiphy.unige.ch
After IVR, the population is distributed among the six isoenergetic states according to the law of statistical thermodynamics. The net result of IVR is the establishment of an intramolecular vibrational temperature. Because of IVR, specific bond breaking through frequency selective multiphoton IR excitation is very inefficient. After this process, the molecular temperature can in principle be very high. For example, after redistribution of $1 \mathrm{eV}$ excess energy, the temperature of a molecule such as benzene is of the order of $1000 \mathrm{~K}$ ! In the condensed phase, such a high temperature is not reached because of VC. Indeed, the hot molecule is in thermal contact with the environment, which is typically at $300 \mathrm{~K}$, and therefore cools down. In Fig. 1, this process corresponds to vertical transitions.
In the condensed phase, VR is ultrafast and occurs on the $10^{-14}-10^{-11} \mathrm{~s}$ timescale. Therefore, for a long time, the dynamics of this process have not been experimentally accessible. With the recent advent of new laser sources producing pulses as short as a few tens of fs, it is now possible to design experiments to investigate VR in the condensed phase. At the moment, our knowledge of these processes is still very sparse. However, it is of paramount importance to understand the details of these energy dissipation phenomena, which are at the origin of the photostability of synthetic and natural pigments and even of essential molecules like nucleic acids. Moreover, photoinduced reactions such as electron transfer [1] or isomerization [2] can occur on a similar timescale. Understanding VR may open

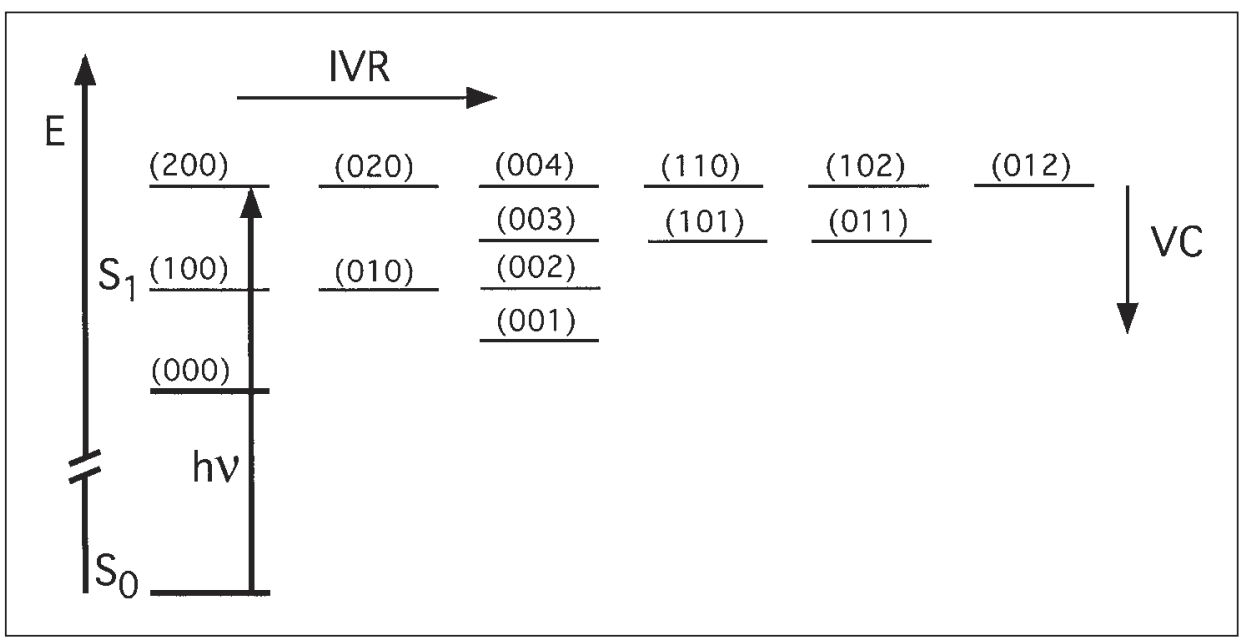

Fig. 1. Energy level scheme for a triatomic molecules with two vibrational modes of the same frequency (modes 1 and 2), and one mode of half that frequency (mode 3). Only mode 1 is Franck-Condon active for optical transition from $\mathrm{S}_{0}$. 
interesting perspectives for influencing or even controlling the dynamics of these ultrafast photochemical reactions.

For these reasons, we have recently started an investigation of VR dynamics of photoexcited molecules, focusing particularly on the influence of solute-solvent interactions on the efficiency of this process. The molecules that we are studying are the perylene derivatives shown in Fig. 2. Depending on the type of solvent, it is possible to tune the strength of the solute-solvent interaction. For example, the weakest interaction is realized with $\mathrm{Pe}$ in hexane, while the strongest one can be expected with $\mathrm{PeOH}$ in alcohols. Although there have been several investigations on VR dynamics over the past years [2-5], no such systematic study has been reported.

The VR dynamics is extracted from time-resolved fluorescence measurements. Fig. 3 shows the $\mathrm{S}_{0}-\mathrm{S}_{1}$ absorption and fluorescence spectra of $\mathrm{PeCN}$ in acetonitrile (ACN). The vibronic structure is dominated by the Franck-Condon active ring breathing mode. Fig. 4 shows the early fluorescence dynamics of $\mathrm{PeCN}$ in $\mathrm{ACN}$ at different wavelengths after excitation with a $100 \mathrm{fs}$ pulse at $400 \mathrm{~nm}$. These measurements were performed using fluorescence up-conversion, which is a pump-probe technique with a time resolution essentially limited by the duration of the laser pulses. At wavelengths located at the wings of the fluorescence band, a fast decay with a time constant of 700 fs can be observed. After this initial decay, the fluorescence intensity decreases exponentially with a time constant of $4.5 \mathrm{~ns}$, which corresponds to the excited state lifetime of PeCN. At wavelengths located in the center of the fluorescence band, the early fluorescence intensity exhibits a rise, which can be reasonably reproduced by a single exponential function with a 700 fs time constant.

These single wavelength time profiles can be used to reconstruct time-resolved fluorescence spectra. It appears that these early dynamics correspond to a narrowing of the emission band that can be ascribed to VR. Other processes like solvation dynamics can be excluded because the electric dipole moment of PeCN does not vary upon excitation. This is precisely a significant advantage of these perylene derivatives, compared to other polar molecules investigated so far, like $p$-nitroaniline [4], where the VR dynamics had to be disentangled from that of other processes like solvation that occur in the same timescale and lead to spectral dynamics as well.

For the non-polar Pe in the non-polar solvent toluene, we have observed a similar but slower spectral narrowing. In this case,

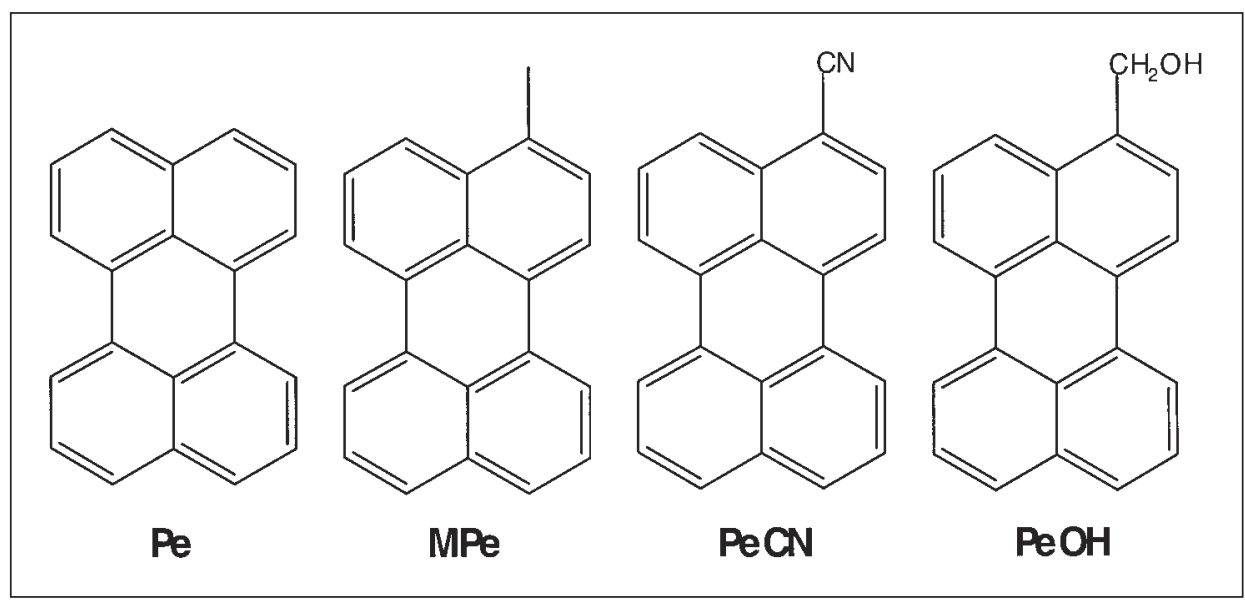

Fig. 2. Perylene derivatives

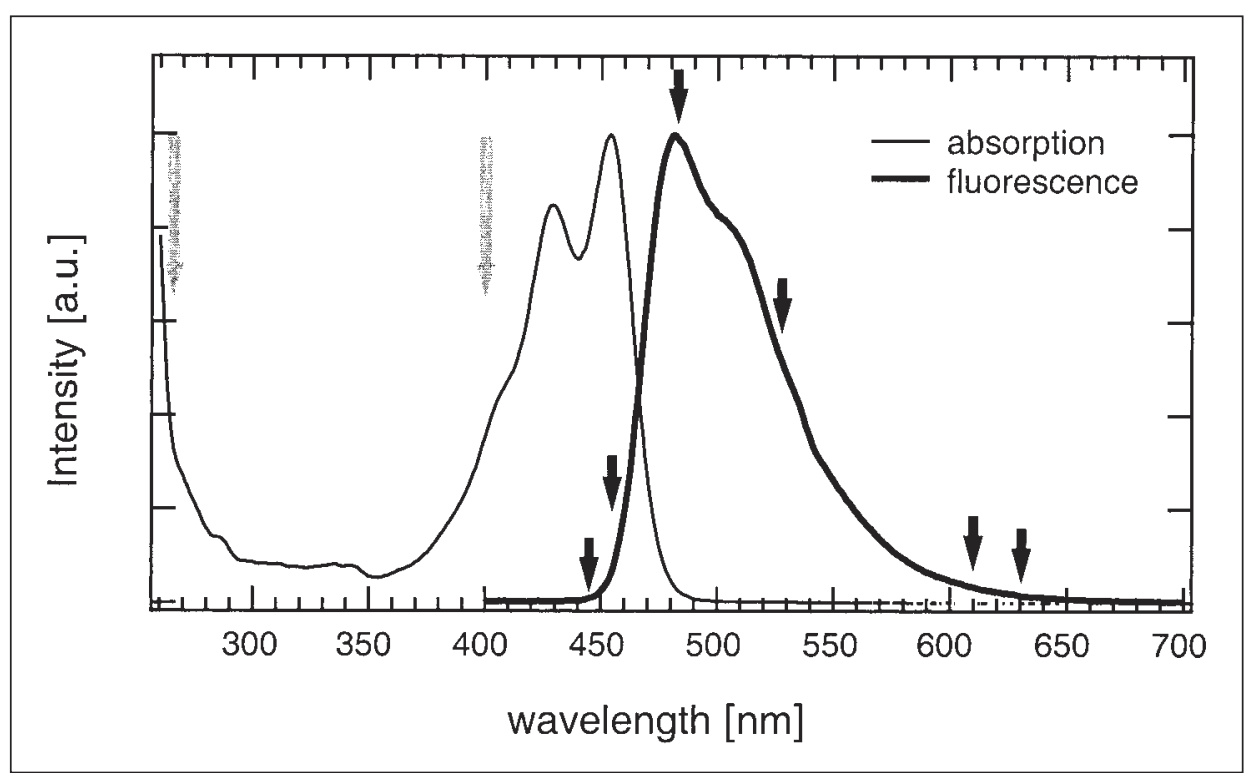

Fig. 3. Absorption and fluorescence spectra of PeCN in ACN. The vertical arrows represent the excitation (grey) and detection (black) wavelengths.

a time constant of $2.4 \mathrm{ps}$ was obtained. This narrowing can be ascribed to $\mathrm{VC}$. VC time constants of the order of $10 \mathrm{ps}$ have been reported for molecules like azulene or stilbene in solution [3]. For $p$-nitroaniline in water, a VC time constant of 640 fs was found [4]. This fast cooling was ascribed to the strength of the solute-solvent coupling via $\mathrm{H}$-bonding. The three-fold acceleration of VC by going from Pe/toluene to PeCN/ $\mathrm{ACN}$ can also be interpreted as an enhanced solute-solvent coupling via the dipole-dipole interaction. Of course, measurements with $\mathrm{Pe}$ in $\mathrm{ACN}$ and $\mathrm{PeCN}$ in toluene have also to be performed to exclude specific solvent effects. Indeed, the thermal conductivity of the solvent can also be expected to affect the VC dynamics.

The same measurements have been performed with PeCN/ACN with $266 \mathrm{~nm}$ excitation. In this case, the excess excitation energy amounts to more than $2 \mathrm{eV}$ ! The early $\mathrm{S}_{1}-\mathrm{S}_{0}$ dynamics exhibit essentially the same wavelength dependence as with $400 \mathrm{~nm}$ excitation. The time constant associated with these dynamics might be slightly shorter, around $600 \pm 100$ fs. This outcome is rather surprising, as one could have expected a more pronounced contribution of the 'hot fluorescence' upon $266 \mathrm{~nm}$ excitation. This result can be interpreted in terms of good thermal conductivity of the solvent. If this was not the case, the first solvent shell around the hot solute molecules would become rather hot as well, and the VC dynamics would be limited by their cooling.

The contribution of IVR to the early fluorescence dynamics observed here cannot be totally excluded. From Fig. 1, it becomes clear that the higher the density of isoenergetic states the faster this process. This implies that the IVR speeds up very quickly with increasing number of atoms, i.e. 


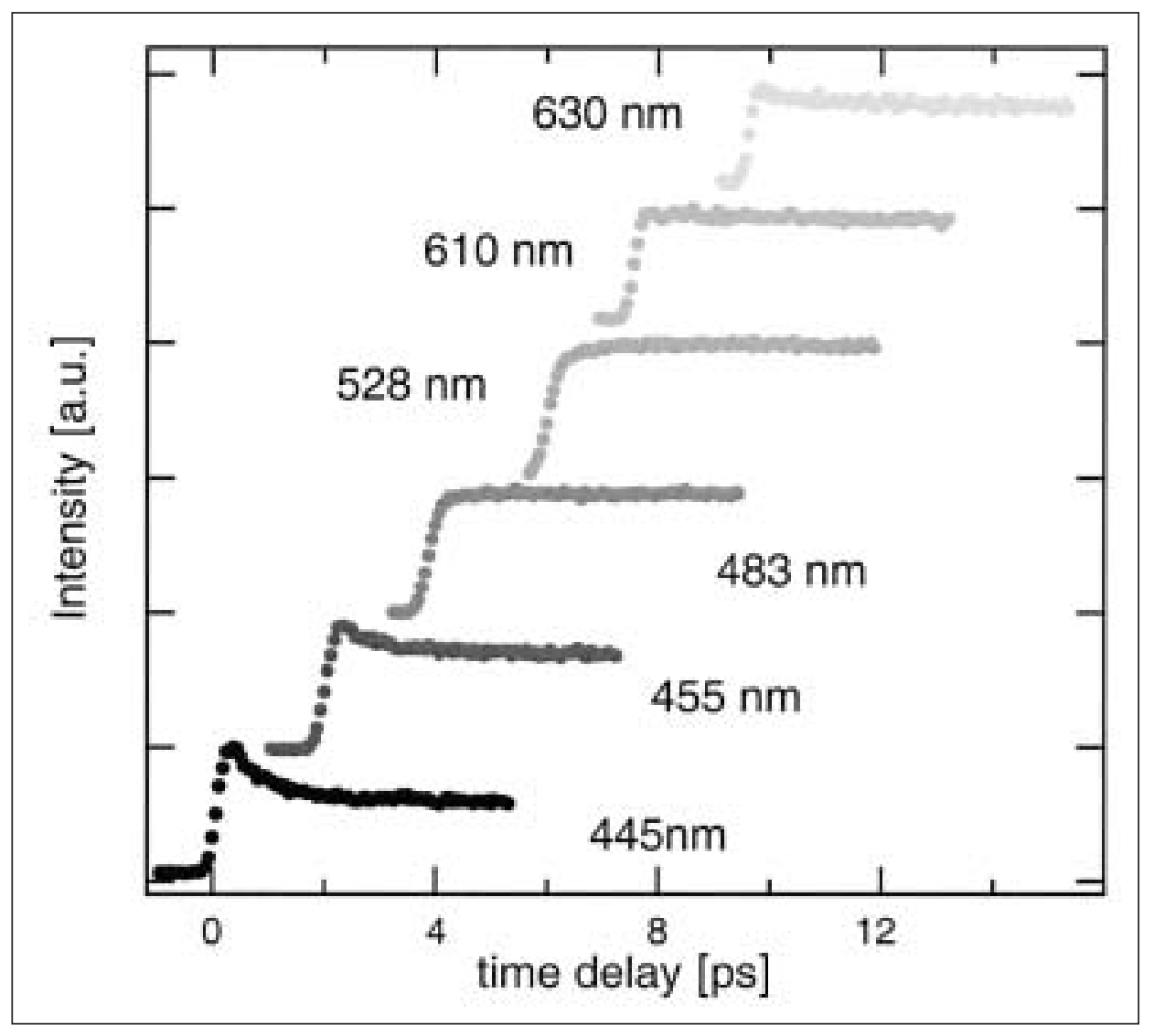

with the size of the molecule. For a relatively small molecule like iodoethane in carbon tetrachloride, an IVR time constant of $1.5 \mathrm{ps}$ has been reported [6]. On the other hand, an upper limit of 100 fs has been reported for the IVR of trans-stilbene in solution [4].

The most unequivocal way to observe the IVR dynamics is to monitor the decay of the fluorescence originating from vibrationally excited states of $S_{1}$. As shown in Fig. 3, the emission of $\mathrm{PeCN}$ around $440 \mathrm{~nm}$ could be due to the $\mathrm{S}_{1}(\mathrm{v}=1) \rightarrow \mathrm{S}_{0}(\mathrm{v}=0)$ transition. This is supported by fluorescence polarization anisotropy measurements. The fluorescence polarization anisotropy $r$ is defined as:

$$
r=\frac{I_{\|}-I_{\perp}}{I_{\|}+2 I_{\perp}}
$$

where $I_{\| \mid}$and $I_{\perp}$ are the fluorescence intensities with a polarization parallel and perpendicular, respectively, to that of the excitation light. The value of $r$ depends on the angle between the transition dipole involved in the excitation process and that involved in the probing process. Of course, $r$ is not constant but decays to zero after ran-
Fig. 4. Early fluorescence dynamics PeCN in $\mathrm{ACN}$ at various wavelengths after excitation at $400 \mathrm{~nm}$.

early $455 \mathrm{~nm}$ emission is very similar to that for the $S_{0}(v=0) \rightarrow S_{1}(v=2)$ transition. Thus, this emission must originate from $\mathrm{S}_{1}(\mathrm{v}=2)$ or $\mathrm{S}_{1}(\mathrm{v}=1)$. In the first case, the fast component should correspond to IVR, while in the latter case, it should be due to VC. The best approach to answer this question is to look for a faster component. If the $0.7 \mathrm{ps}$ is the fastest component, it should most probably correspond to IVR. Of course, to ensure that there are no faster components, the time resolution of the experiment must be of the order of a few tens of fs. This is not the case at the moment, although we hope to build such a set-up in the near future. However, the beginning of an answer can be obtained from our 'low' resolution measurements. Indeed, Fig. 6 shows that the increase of the fluorescence intensity varies substantially from the blue to the red wavelengths. In the blue wavelength, this increase is close to the temporal response of the experiment but it is markedly slower at longer wavelengths. This effect has not been investigated in detail yet, but it indicates rather clearly dynamic features substantially faster than $0.7 \mathrm{ps}$.

The data presented here are just the first results of our investigation of the VR dynamics of large molecules in solution. They show that ultrafast fluorescence up-conversion is well suited for such investigations. We are going to investigate systematically the influence of solute-solvent interaction on the VC dynamics. We also plan to build a new up-conversion set-up with a sufficiently good time resolution to be able to measure faster VR dynamics.

With this, we hope to able to find simple rules that should help to predict the efficiency of heat dissipation of a given solute/ solvent system. 

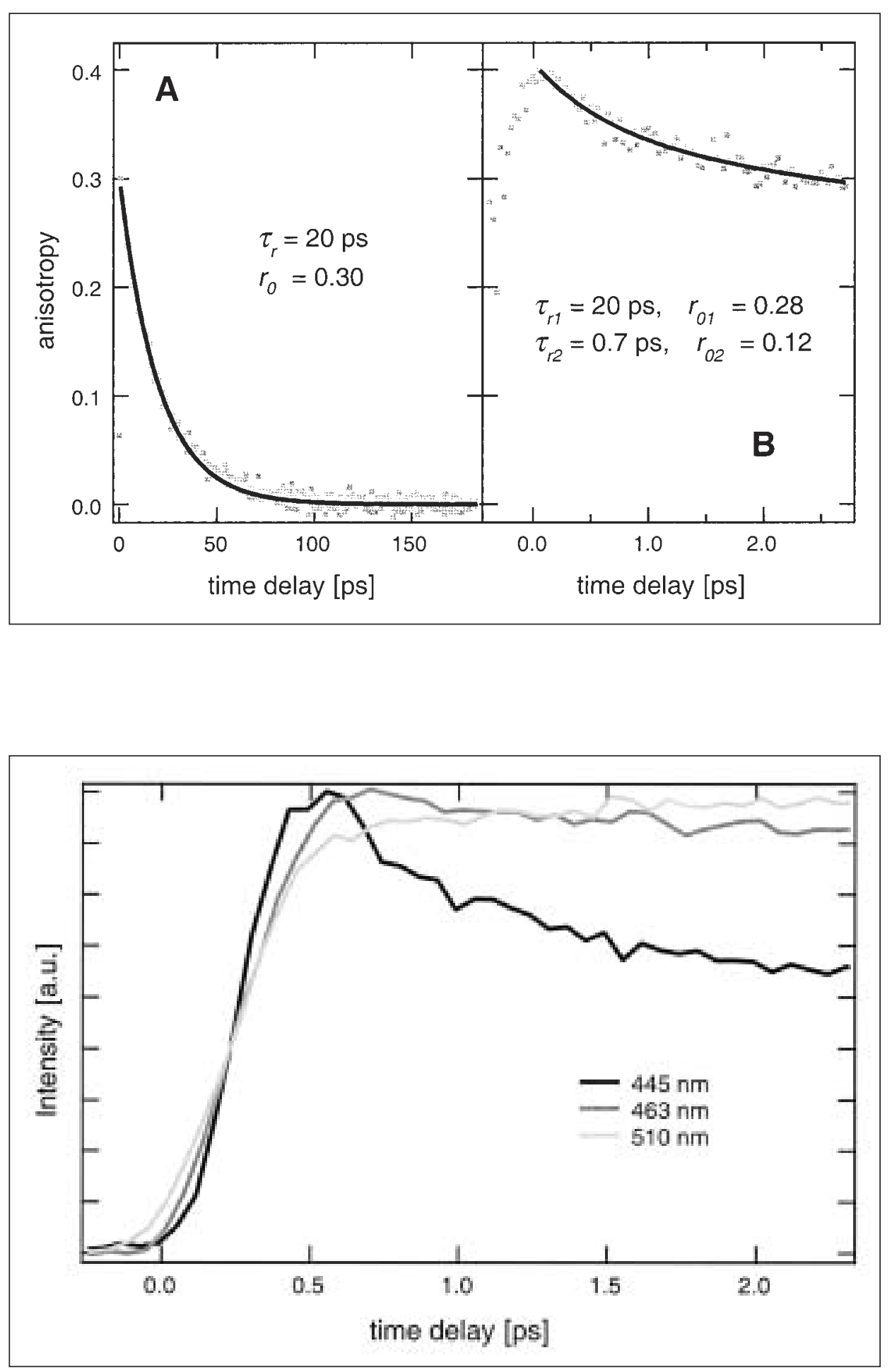

\section{Acknowledgement}

This work is supported by the Fonds National Suisse de la Recherche Scientifique through project Nr. 2000-0632528.00

Received: February 7, 2003

[1] O. Nicolet, E. Vauthey, J. Phys. Chem. A 2002, 206, 5553.

[2] R.J. Sension, T. Repinec, R.M. Hochstrasser, J. Chem. Phys. 1995, 93, 9185.
[3] D. Schwarzer, J. Troe, M. Zerezke, J. Chem.Phys. 1997, 107, 8380.

[4] S. A. Kovalenko, R. Schanz, H. Henning, N.P. Ernsting, J. Chem. Phys. 2001, 115, 3256.

[5] D. D. Dlott, Chem. Phys. 2001, 266, 149; T. Yamaguchi, Y. Kimura, N. Hirota, $J$. Chem. Phys. 2000, 113, 2772.

[6] J. Assmann, A. Charvat, D. Schwarzer, Chem. A 2002, 106, 5197. C. Kappel, K. Luther, B. Abel, J. Phys.
Fig. 5. Decay of the fluorescence polarization anisotropy measured at (A) $510 \mathrm{~nm}$ and (B) $455 \mathrm{~nm}$ (early dynamics) after excitation of $\mathrm{PeCN}$ at $400 \mathrm{~nm}$ in $\mathrm{ACN}$.

Fig. 6. Wavelength dependence of the initial rise of the fluorescence intensity of $\mathrm{PeCN}$ upon $400 \mathrm{~nm}$ excitation. 